\title{
APPROXIMATION ON THE SPHERE BY WEIGHTED FOURIER EXPANSIONS
}

\author{
V. A. MENEGATTO AND A. C. PIANTELLA \\ Received 2 March 2005 and in revised form 12 September 2005
}

The main theme of this paper is the approximation on the sphere by weighted sums of spherical harmonics. We give necessary and sufficient conditions on the weights for convergence in both the continuous and the $L^{p}$ cases. Approximation by spherical convolution is a particular and important case that fits into our setting.

\section{Introduction and basic facts}

A standard procedure to approximate a function $f$ in an inner product space is to consider the Fourier series of the function with respect to an orthogonal system. The basic general results on this topic can be found in many references in the literature, for example, [2, Chapter VIII].

It is well known that even in the case in which $K$ is a closed interval there always exists a function $f$ in $C(K)$ for which the corresponding Fourier series does not converge to $f$ with respect to the uniform norm. Thus, in this and other cases, the common solution is to consider weighted expansions and to study convergence based on the choice of the weights. Here is a list of problems that emerges: how to choose the weights in order to guarantee convergence for every function in the space, to study orders of convergence, how to choose the weights so that the operators given by the truncated Fourier series inherit properties of other known operators, and so forth.

In this paper, we consider some of the problems above in the case when $K=S^{m}$, the unit sphere in $\mathbb{R}^{m+1}$. The focus is on convergence but we intend to study the analysis of convergence orders in a forthcoming paper. For functions defined on $S^{m}$, orthogonality depends upon $d \sigma_{m}$, the usual surface measure on $S^{m}$. The surface area of $S^{m}$ will be written as $\sigma_{m}$. The uniform norm is then given by

$$
\|f\|_{\infty}:=\sup _{x \in S^{m}}|f(x)|, \quad f \in C\left(S^{m}\right)
$$

and as usual, we consider the spaces $L^{p}\left(S^{m}\right):=L^{p}\left(S^{m}, d \sigma_{m}\right), 1 \leq p<\infty$, with norm given by

$$
\|f\|_{p}:=\left(\frac{1}{\sigma_{m}} \int_{S^{m}}|f(x)|^{p} d \sigma_{m}(x)\right)^{1 / p}, \quad f \in L^{p}\left(S^{m}\right) .
$$


Unless stated otherwise, the letter $X$ will denote any of the spaces introduced above and $\|\cdot\|_{X}$ the corresponding norm. Orthogonality is then related to the inner product of $L^{2}\left(S^{m}\right)$

$$
\langle f, g\rangle_{2}=\frac{1}{\sigma_{m}} \int_{S^{m}} f \bar{g} d \sigma_{m}, \quad f, g \in L^{2}\left(S^{m}\right) .
$$

We will write $P\left(S^{m}\right)$ to denote the space formed by polynomials in $m+1$ variables restricted to $S^{m}$. The set $\mathscr{H}_{k}\left(S^{m}\right)$ of all spherical harmonics of degree $k$ in $m+1$ variables joint with the zero polynomial is a subspace of $P\left(S^{m}\right)$. Let $\left\{Y_{k 1}, Y_{k 2}, \ldots, Y_{k N(m, k)}\right\}$ be an orthonormal basis of $\mathscr{H}_{k}\left(S^{m}\right)$. To every function in any of the spaces above, we can associate the Fourier series

$$
f \sim \sum_{k=0}^{\infty} \sum_{l=1}^{N(m, k)} \hat{f}(k, l) Y_{k l}
$$

in which the Fourier coefficient $\hat{f}(k, l)$ is given by

$$
\hat{f}(k, l)=\int_{S^{m}} f \overline{Y_{k l}} d \sigma_{m} .
$$

The setting introduced above is, up to normalization, the same used in many standard references on analysis on the sphere. We refer the reader to $[1,4,7,11,12,13,14]$, where the Addition Formula

$$
\sum_{l=1}^{N(m, k)} Y_{k l}(x) \overline{Y_{k l}(y)}=\frac{N(m, k)}{\sigma_{m}} P_{k}^{m}(\langle x, y\rangle), \quad x, y \in S^{m},
$$

the Funk-Hecke Formula

$$
\int_{S^{m}} K(\langle x, y\rangle) Y_{k}(y) d \sigma_{m}(y)=a_{k}^{m}(K) Y_{k}(x), \quad x \in S^{m},
$$

and other results can be found. In (1.6) and (1.7), $P_{k}^{m}$ is the Legendre polynomial of degree $k$ associated to the dimension $m+1, K:[-1,1] \rightarrow \mathbb{C}$ is an integrable function, $Y_{k} \in \mathscr{H}_{k}\left(S^{m}\right)$, and

$$
a_{k}^{m}(K):=\sigma_{m-1} \int_{-1}^{1} K(t) P_{k}^{m}(t)\left(1-t^{2}\right)^{(m-2) / 2} d t
$$

We will deal with approximations of a given function $f$ in $X$ by a sequence $\left\{T_{n}(f)\right\}_{n \in \mathbb{N}}$, in which the operators $T_{n}: X \rightarrow X, n \in \mathbb{N}:=\{0,1, \ldots\}$, are defined by weighted orthogonal Fourier sums of the form

$$
T_{n}(f)=\frac{1}{\sigma_{m}} \sum_{k=0}^{n} \sum_{l=1}^{N(m, k)} a_{k l}(n) \hat{f}(k, l) Y_{k l}, \quad f \in X,
$$

the weights $a_{k l}(n), n, k=0,1, \ldots, l=1,2, \ldots, N(m, k)$ being all real. In Section 2 , we deduce some basic properties of the operators $T_{n}$, including the computation of their norm 
in the cases $X=C\left(S^{m}\right)$ and $X=L^{1}\left(S^{m}\right)$. In Section 3, we find necessary and sufficient conditions in order that $\left\{T_{n}\right\}_{n \in \mathbb{N}}$ be an approximate identity in $X$. Among other things, this implies that $T_{n}(f)$ converges to $f$ in the norm of $X$, for every $f \in X$. In Section 4 , restricting ourselves to the case $X=C\left(S^{m}\right)$, we introduce the notion of localized approximate identity and study corresponding approximation properties.

\section{The operator $T_{n}$}

In this section, we present some basic properties of the operator $T_{n}$, some of independent interest, other to be used in the subsequent sections. That includes to decide when $T_{n}$ is of convolution type and the computation of its norm in some cases.

The orthonormality of the spherical harmonics yields

$$
T_{n}\left(Y_{k l}\right)= \begin{cases}a_{k l}(n) Y_{k l}, & k \leq n \\ 0, & k>n\end{cases}
$$

The linearity of $T_{n}$ reveals that

$$
T_{n}(q)=\sum_{k=0}^{M} \sum_{l=1}^{N(m, k)} a_{k l}(n) r_{k l} Y_{k l}, \quad n \geq M
$$

whenever

$$
q=\sum_{\mu=0}^{M} \sum_{\nu=1}^{N(m, \mu)} r_{\mu \nu} Y_{\mu \nu}, \quad r_{\mu \nu} \in \mathbb{C}
$$

Writing $T_{n} f(x):=\left(T_{n}(f)\right)(x)$ and appealing to (1.5), we deduce that

$$
\begin{aligned}
T_{n} f(x) & =\frac{1}{\sigma_{m}} \sum_{k=0}^{n} \sum_{l=1}^{N(m, k)} a_{k l}(n)\left(\int_{S^{m}} f(y) \overline{Y_{k l}(y)} d \sigma_{m}(y)\right) Y_{k l}(x) \\
& =\frac{1}{\sigma_{m}} \int_{S^{m}} f(y) \sum_{k=0}^{n} \sum_{l=1}^{N(m, k)} a_{k l}(n) Y_{k l}(x) \overline{Y_{k l}(y)} d \sigma_{m}(y) .
\end{aligned}
$$

Thus, we have proved the following representation formula.

Theorem 2.1. If $f \in X$ then

$$
T_{n} f(x)=\frac{1}{\sigma_{m}} \int_{S^{m}} K_{n}(x, y) f(y) d \sigma_{m}(y), \quad x \in S^{m},
$$

in which

$$
K_{n}(x, y)=\sum_{k=0}^{n} \sum_{l=1}^{N(m, k)} a_{k l}(n) Y_{k l}(x) \overline{Y_{k l}(y)}, \quad x, y \in S^{m} .
$$

Formula (2.6) defines a kernel having an expression very close to that on the left-hand side of the Addition Formula. Since that formula defines a bi-zonal polynomial kernel 
324 Approximation on the sphere by weighted Fourier expansions

and this type of kernel defines spherical convolution operators on $X$, the goal in the next two results will thus be to verify when $T_{n}$ is an operator of convolution type.

LeMmA 2.2. The kernel $K_{n}$ is bi-zonal if and only if $a_{k 1}(n)=a_{k 2}(n)=\cdots=a_{k N(m, k)}(n)$, $k=0,1, \ldots, n$.

Proof. If $a_{k 1}(n)=a_{k 2}(n)=\cdots=a_{k N(m, k)}(n), k=0,1, \ldots, n$, the Addition Formula implies that

$$
K_{n}(x, y)=\sum_{k=0}^{n} a_{k 1}(n) \frac{N(m, k)}{\sigma_{m}} P_{k}^{m}(\langle x, y\rangle):=L_{n}(\langle x, y\rangle), \quad x, y \in S^{m},
$$

for some function $L_{n}$, that is, $K_{n}$ is bi-zonal. Conversely, fix $k \in\{0,1, \ldots, n\}$ and $l$ in the set $\{1,2, \ldots, N(m, k)\}$. If $K_{n}(x, y)=L_{n}(\langle x, y\rangle)$ for some function $L_{n},(1.7)$ yields

$$
\int_{S^{m}} K_{n}(x, y) Y_{k l}(y) d \sigma_{m}(y)=\int_{S^{m}} L_{n}(\langle x, y\rangle) Y_{k l}(y) d \sigma_{m}(y)=a_{k}^{m}\left(L_{n}\right) Y_{k l}(x), \quad x \in S^{m},
$$

while the previous theorem and relation (2.1) imply that

$$
\int_{S^{m}} K_{n}(x, y) Y_{k l}(y) d \sigma_{m}(y)=\sigma_{m} a_{k l}(n) Y_{k l}(x), \quad x \in S^{m}
$$

Thus,

$$
a_{k}^{m}\left(L_{n}\right) Y_{k l}(x)=\sigma_{m} a_{k l}(n) Y_{k l}(x), \quad x \in S^{m} .
$$

Since $Y_{k l} \not \equiv 0$, we conclude that $a_{k l}(n)=a_{k}^{m}\left(L_{n}\right) / \sigma_{m}, l=1,2, \ldots, N(m, k)$.

Let $K:[-1,1] \rightarrow \mathbb{C}$ be a function such that

$$
\int_{-1}^{1}|K(t)|\left(1-t^{2}\right)^{(m-2) / 2} d t<\infty
$$

The spherical convolution defined by $K$ is the operator $f \in X \mapsto T_{K}(f) \in X$ given by

$$
T_{K}(f)(x)=\frac{1}{\sigma_{m}} \int_{S^{m}} K(\langle x, y\rangle) f(y) d \sigma_{m}(y), \quad x \in S^{m} .
$$

Basic properties of this operator along with some information about bi-zonal kernels are to be found in $[4,7,8,14]$ and the references therein. We adopt here the most common notation for the convolution operator, that is, $T_{K}(f)=K * f$.

THEOREM 2.3. The operator $T_{n}$ is of convolution type if and only if $K_{n}$ is bi-zonal. 
Proof. If $K_{n}(x, y)=L_{n}(\langle x, y\rangle), x, y \in S^{m}$, for some $L_{n}$, then $T_{n}(f)=L_{n} * f, f \in X$, by Theorem 2.1. Conversely, if $T_{n}(f)=L_{n} * f, f \in X$, for some $L_{n}$ then

$$
\int_{S^{m}} K_{n}(x, y) f(y) d \sigma_{m}(y)=\int_{S^{m}} L_{n}(\langle x, y\rangle) f(y) d \sigma_{m}(y), \quad x \in S^{m}, f \in X,
$$

that is,

$$
\int_{S^{m}}\left[K_{n}(x, y)-L_{n}(\langle x, y\rangle)\right] f(y) d \sigma_{m}(y)=0, \quad x \in S^{m}, f \in X .
$$

In particular,

$$
\int_{S^{m}}\left[K_{n}(x, y)-L_{n}(\langle x, y\rangle)\right] Y_{k l}(y) d \sigma_{m}(y)=0, \quad x \in S^{m}, k \in \mathbb{N}, l=1,2, \ldots, N(m, k) .
$$

Since $\left\{Y_{k l}: k \in \mathbb{N}, l=1, \ldots, N(m, k)\right\}$ is a complete orthonormal subset of $L^{2}\left(S^{m}\right)$, it follows that

$$
K_{n}(x, y)-L_{n}(\langle x, y\rangle)=0 \quad \text { a.e. }
$$

This leads to $K_{n}(x, y)=L_{n}(\langle x, y\rangle), x, y \in S^{m}$, because $K_{n}$ is polynomial.

Next, we introduce some notation. Given a kernel $K: S^{m} \times S^{m} \rightarrow \mathbb{C}$, we will write $K^{x}$ e $K^{y}$ to denote the functions $y \in S^{m} \mapsto K(x, y)$ and $x \in S^{m} \mapsto K(x, y)$, respectively. It is easily seen that these two functions are in $L^{1}\left(S^{m}\right)$ when $K$ is polynomial. In just one step of the proof of Theorem 2.4 below we use the space $L^{\infty}\left(S^{m}\right)$ as defined in [3, page 184].

Theorem 2.4. An upper bound for $\left\|T_{n}\right\|_{X}$ is $\sup \left\{\left\|K_{n}^{x}\right\|_{1}: x \in S^{m}\right\}$.

Proof. First we consider the case $X=C\left(S^{m}\right)$. If $f \in X$, Theorem 2.1 implies that

$$
\left|T_{n} f(x)\right| \leq \frac{1}{\sigma_{m}} \int_{S^{m}}\left|K_{n}(x, y) f(y)\right| d \sigma_{m}(y) \leq\left\|K_{n}^{x}\right\|_{1}\|f\|_{\infty}, \quad x \in S^{m} .
$$

Hence,

$$
\left\|T_{n}(f)\right\|_{\infty} \leq\|f\|_{\infty} \sup \left\{\left\|K_{n}^{x}\right\|_{1}: x \in S^{m}\right\}, \quad f \in X,
$$

and, consequently,

$$
\left\|T_{n}\right\|_{C\left(S^{m}\right)} \leq \sup \left\{\left\|K_{n}^{x}\right\|_{1}: x \in S^{m}\right\} .
$$


326 Approximation on the sphere by weighted Fourier expansions

Next, we consider the case $X=L^{p}\left(S^{m}\right)$. If $f \in X$ then

$$
\begin{aligned}
\left\|T_{n}(f)\right\|_{p}^{p} & =\frac{1}{\sigma_{m}} \int_{S^{m}}\left|T_{n} f(x)\right|^{p} d \sigma_{m}(x) \\
& =\frac{1}{\sigma_{m}} \int_{S^{m}}\left|\frac{1}{\sigma_{m}} \int_{S^{m}} K_{n}(x, y) f(y) d \sigma_{m}(y)\right|^{p} d \sigma_{m}(x) \\
& \leq \frac{1}{\sigma_{m}} \int_{S^{m}}\left[\frac{1}{\sigma_{m}} \int_{S^{m}}\left|K_{n}(x, y) f(y)\right| d \sigma_{m}(y)\right]^{p} d \sigma_{m}(x) .
\end{aligned}
$$

Since $\left(K_{n}^{x}\right)^{1 / p} f \in L^{p}\left(S^{m}\right), x \in S^{m}$ and $\left(K_{n}^{x}\right)^{1 / p^{\prime}} \in L^{p^{\prime}}\left(S^{m}\right), x \in S^{m}$ ( $p^{\prime}$ is the conjugate exponent of $p$ ), Hölder's inequality implies that

$$
\begin{aligned}
{\left[\frac{1}{\sigma_{m}} \int_{S^{m}}\left|K_{n}(x, y) f(y)\right| d \sigma_{m}(y)\right]^{p} } & \leq\left\|\left(K_{n}^{x}\right)^{1 / p^{\prime}}\right\|_{p^{\prime}}^{p}\left\|\left(K_{n}^{x}\right)^{1 / p} f\right\|_{p}^{p} \\
& =\frac{1}{\sigma_{m}}\left\|K_{n}^{x}\right\|_{1}^{p / p^{\prime}} \int_{S^{m}}\left|K_{n}(x, y)\right||f(y)|^{p} d \sigma_{m}(y) .
\end{aligned}
$$

Picking $x_{0} \in S^{m}$ such that

$$
\sup \left\{\left\|K_{n}^{x}\right\|_{1}: x \in S^{m}\right\}=\left\|K_{n}^{x_{0}}\right\|_{1}
$$

and using Fubini's theorem, we obtain

$$
\begin{aligned}
\left\|T_{n}(f)\right\|_{p}^{p} & \leq \frac{1}{\sigma_{m}^{2}} \int_{S^{m}}\left\|K_{n}^{x}\right\|_{1}^{p / p^{\prime}} \int_{S^{m}}\left|K_{n}(x, y)\right||f(y)|^{p} d \sigma_{m}(y) d \sigma_{m}(x) \\
& \leq \frac{1}{\sigma_{m}^{2}}\left\|K_{n}^{x_{0}}\right\|_{1}^{p / p^{\prime}} \int_{S^{m}} \int_{S^{m}}\left|K_{n}(x, y)\right||f(y)|^{p} d \sigma_{m}(y) d \sigma_{m}(x) \\
& =\frac{1}{\sigma_{m}}\left\|K_{n}^{x_{0}}\right\|_{1}^{p / p^{\prime}} \int_{S^{m}}\left(\frac{1}{\sigma_{m}} \int_{S^{m}}\left|\overline{K_{n}(y, x)}\right| d \sigma_{m}(x)\right)|f(y)|^{p} d \sigma_{m}(y) .
\end{aligned}
$$

Thus,

$$
\left\|T_{n}(f)\right\|_{p}^{p} \leq \frac{1}{\sigma_{m}}\left\|K_{n}^{x_{0}}\right\|_{1}^{p / p^{\prime}} \int_{S^{m}}\left\|K_{n}^{y}\right\|_{1}|f(y)|^{p} d \sigma_{m}(y) \leq\left\|K_{n}^{x_{0}}\right\|_{1}^{p}\|f\|_{p}^{p}
$$

whence

$$
\left\|T_{n}\right\|_{X} \leq \sup \left\{\left\|K_{n}^{x}\right\|_{1}: x \in S^{m}\right\}
$$

The proof is complete.

We would like to observe that the second half of the proof above is very close to that of [3, Theorem 6.18]. The upper bound given above coincides with $\left\|T_{n}\right\|_{X}$ in at least two cases. 
Theorem 2.5. For $X=C\left(S^{m}\right)$ and $X=L^{1}\left(S^{m}\right)$, it holds

$$
\left\|T_{n}\right\|_{X}=\sup \left\{\left\|K_{n}^{x}\right\|_{1}: x \in S^{m}\right\}
$$

Proof. Due to Theorem 2.4, we need to prove that

$$
\left\|T_{n}\right\|_{X} \geq \sup \left\{\left\|K_{n}^{x}\right\|_{1}: x \in S^{m}\right\}
$$

Using the formula [3, page 189]

$$
\|f\|_{1}=\sup \left\{\left|\frac{1}{\sigma_{m}} \int_{S^{m}} f \bar{g} d \sigma_{m}\right|:\|g\|_{\infty}=1\right\}, \quad f \in L^{1}\left(S^{m}\right),
$$

we obtain

$$
\begin{aligned}
\left\|K_{n}^{x}\right\|_{1} & =\sup \left\{\left|\frac{1}{\sigma_{m}} \int_{S^{m}} K_{n}(x, y) \overline{f(y)} d \sigma_{m}(y)\right|:\|f\|_{\infty}=1\right\} \\
& =\sup \left\{\left|\frac{1}{\sigma_{m}} \int_{S^{m}} K_{n}(x, y) f(y) d \sigma_{m}(y)\right|:\|\bar{f}\|_{\infty}=1\right\} \\
& =\sup \left\{\left|T_{n} f(x)\right|:\|f\|_{\infty}=1\right\} \\
& \leq \sup \left\{\left\|T_{n}(f)\right\|_{\infty}:\|f\|_{\infty}=1\right\} \\
& =\left\|T_{n}\right\|_{C\left(S^{m}\right)}, \quad x \in S^{m},
\end{aligned}
$$

and, consequently,

$$
\sup \left\{\left\|K_{n}^{x}\right\|_{1}: x \in S^{m}\right\} \leq\left\|T_{n}\right\|_{C\left(S^{m}\right)} .
$$

To finish the proof, first observe that

$$
\begin{aligned}
\int_{S^{m}} T_{n} f(y) \overline{g(y)} d \sigma_{m}(y) & =\frac{1}{\sigma_{m}} \int_{S^{m}} \sum_{k=0}^{n} \sum_{l=1}^{N(m, k)} a_{k l}(n) \hat{f}(k, l) Y_{k l}(y) \overline{g(y)} d \sigma_{m}(y) \\
& =\int_{S^{m}}\left(\frac{1}{\sigma_{m}} \sum_{k=0}^{n} \sum_{l=1}^{N(m, k)} a_{k l}(n) \overline{\hat{g}(k, l) Y_{k l}(y)}\right) f(y) d \sigma_{m}(y) \\
& =\overline{\int_{S^{m}} T_{n} g(y) \overline{f(y)} d \sigma_{m}(y)}, \quad f \in L^{1}\left(S^{m}\right), g \in C\left(S^{m}\right) .
\end{aligned}
$$

Finally, using the formula [3, page 223]

$$
\|f\|_{\infty}=\sup \left\{\left|\frac{1}{\sigma_{m}} \int_{S^{m}} f \bar{g} d \sigma_{m}\right|:\|g\|_{1}=1\right\},
$$


328 Approximation on the sphere by weighted Fourier expansions

we obtain

$$
\begin{aligned}
\left\|T_{n}\right\|_{L_{1}\left(S^{m}\right)} & =\sup \left\{\left\|T_{n} f\right\|_{1}:\|f\|_{1}=1\right\} \\
& =\sup _{\|f\|_{1}=1}\left(\sup \left\{\left|\frac{1}{\sigma_{m}} \int_{S^{m}} T_{n} f(y) \overline{g(y)} d \sigma_{m}(y)\right|:\|g\|_{\infty}=1\right\}\right) \\
& =\sup _{\|f\|_{1}=1}\left(\sup \left\{\left|\frac{1}{\sigma_{m}} \int_{S^{m}} T_{n} g(y) \overline{f(y)} d \sigma_{m}(y)\right|:\|g\|_{\infty}=1\right\}\right) \\
& =\sup _{\|g\|_{\infty}=1}\left(\sup \left\{\left|\frac{1}{\sigma_{m}} \int_{S^{m}} T_{n} g(y) \overline{f(y)} d \sigma_{m}(y)\right|:\|f\|_{1}=1\right\}\right) \\
& =\sup _{n}\left\{\left\|T_{n}(g)\right\|_{\infty}:\|g\|_{\infty}=1\right\} \\
& =\left\|T_{n}\right\|_{C\left(S^{m}\right)} .
\end{aligned}
$$

This completes the proof of the theorem.

\section{Approximate identities}

In this section, keeping the notation in (1.9), we will search for conditions in order that the following approximation property holds:

$$
\lim _{n \rightarrow \infty}\left\|T_{n}(f)-f\right\|_{X}=0, \quad f \in X
$$

As usual, a sequence $\left\{T_{n}\right\}_{n \in \mathbb{N}}$ satisfying (3.1) is called an approximate identity in $X$. We refer the reader to [8] and some references therein, for nice examples of approximate identities in $X$, including examples involving spherical convolution, spherical shifting, and so forth. Since spherical convolution operators belong to the class of operators we are dealing with here, many results in this section may be considered as generalizations of those corresponding to approximation by spherical convolution. For results dealing with approximate identities and spherical convolution, see $[5,6,8,9,10]$.

Theorem 3.1. If $\left\{T_{n}\right\}_{n \in \mathbb{N}}$ is an approximate identity in $X$ then the following properties hold:

(i) There exists a positive constant $C$ such that $\left\|T_{n}(f)\right\|_{X} \leq C\|f\|_{X}, n \in \mathbb{N}, f \in X$;

(ii) $\lim _{n \rightarrow \infty} a_{k l}(n)=1, k \in \mathbb{N}, l=1,2, \ldots, N(m, k)$.

Proof. Assume $\left\{T_{n}\right\}_{n \in \mathbb{N}}$ is an approximate identity in $X$. Since every sequence $\left\{T_{n}(f)\right\}_{n \in \mathbb{N}}, f \in X$, is bounded, the Uniform Boundedness Principle implies that

$$
\left\|T_{n}(f)\right\|_{X} \leq C\|f\|_{X}, \quad f \in X, n \in \mathbb{N}
$$

for some nonnegative constant $C$. If $C=0$ then $T_{n}(f)=0, f \in X, n \in \mathbb{N}$, whence

$$
\|f\|_{X}=\lim _{n \rightarrow \infty}\left\|T_{n}(f)-f\right\|_{X}=0, \quad f \in X
$$


a clear contradiction. Thus, $C>0$ and (i) is proved. To prove (ii) fix $k \in \mathbb{N}$ and $l \in$ $\{1,2, \ldots, N(m, k)\}$. Since

$$
T_{n} Y_{k l}(y)=a_{k l}(n) Y_{k l}(y), \quad y \in S^{m}, n \geq k,
$$

it follows that

$$
\lim _{n \rightarrow \infty} T_{n} Y_{k l}(y)=\left(\lim _{n \rightarrow \infty} a_{k l}(n)\right) Y_{k l}(y), \quad y \in S^{m}
$$

We split the proof in two cases. If $X=C\left(S^{m}\right)$, our assumption on $\left\{T_{n}\right\}_{n \in \mathbb{N}}$ implies that

$$
\lim _{n \rightarrow \infty} T_{n} Y_{k l}(y)=Y_{k l}(y), \quad y \in S^{m}
$$

Since $Y_{k l} \not \equiv 0$, we can choose $y_{0} \in S^{m}$ such that $Y_{k l}\left(y_{0}\right) \neq 0$. Thus, (3.5) and (3.6) lead to

$$
Y_{k l}\left(y_{0}\right)=\left(\lim _{n \rightarrow \infty} a_{k l}(n)\right) Y_{k l}\left(y_{0}\right)
$$

If $X=L^{p}\left(S^{m}\right), 1 \leq p<\infty$, a similar procedure leads to

$$
Y_{k l}(y)=\lim _{n \rightarrow \infty} T_{n} Y_{k l}(y)=\left(\lim _{n \rightarrow \infty} a_{k l}(n)\right) Y_{k l}(y), \quad y \in S^{m} \backslash \Lambda
$$

where $\sigma_{m}(\Lambda)=0$. Taking $y_{1} \in S^{m} \backslash \Lambda$ such that $Y_{k l}\left(y_{1}\right) \neq 0$, as we certainly can, we conclude that

$$
Y_{k l}\left(y_{1}\right)=\left(\lim _{n \rightarrow \infty} a_{k l}(n)\right) Y_{k l}\left(y_{1}\right)
$$

In both cases the conclusion is $\lim _{n \rightarrow \infty} a_{k l}(n)=1$.

Next, we search for some converse results.

THeOREM 3.2. Let $f \in C\left(S^{m}\right)$ be a function fulfilling the following condition: if $q \in P\left(S^{m}\right)$ then there exists a constant $C:=C(f-q) \geq 0$ such that $\left\|T_{n}(f-q)\right\|_{\infty} \leq C(f-q) \| f-$ $q \|_{\infty}, n \in \mathbb{N}$. If $\lim _{n \rightarrow \infty} a_{k l}(n)=1, k \in \mathbb{N}, l=1,2, \ldots, N(m, k)$ then $\lim _{n \rightarrow \infty}\left\|T_{n}(f)-f\right\|_{\infty}=0$.

Proof. Let $\epsilon>0$. We use the Weierstrass approximation theorem to select $q \in P\left(S^{m}\right)$ such that $\|f-q\|_{\infty}<\epsilon$ and write $q$ in the form

$$
q=\sum_{k=0}^{M} \sum_{l=1}^{N(m, k)} r_{k l} Y_{k l}, \quad r_{k l} \in \mathbb{C}, k=0,1, \ldots, M, l=1,2, \ldots, N(m, k)
$$


Since

$$
T_{n}(q)=\sum_{k=0}^{M} \sum_{l=1}^{N(m, k)} a_{k l}(n) r_{k l} Y_{k l}, \quad n \geq M,
$$

defining

$$
\begin{aligned}
& B_{1}:=\max \left\{\left|r_{k l}\right|: k=0,1, \ldots, M, l=1,2, \ldots, N(m, k)\right\}, \\
& B_{2}:=\max \left\{\left\|Y_{k l}\right\|_{\infty}: k=0,1, \ldots, M, l=1,2, \ldots, N(m, k)\right\},
\end{aligned}
$$

it is clear that

$$
\begin{aligned}
\left|T_{n} q(y)-q(y)\right| & =\left|\sum_{k=0}^{M} \sum_{l=1}^{N(m, k)}\left(a_{k l}(n)-1\right) r_{k l} Y_{k l}(y)\right| \\
& \leq \sum_{k=0}^{M} \sum_{l=1}^{N(m, k)}\left|a_{k l}(n)-1\right|\left|r_{k l}\right||| Y_{k l}||_{\infty} \\
& \leq B_{1} B_{2} \sum_{k=0}^{M} \sum_{l=1}^{N(m, k)}\left|a_{k l}(n)-1\right|, \quad y \in S^{m}, n \geq M .
\end{aligned}
$$

Consequently,

$$
\left\|T_{n}(q)-q\right\|_{\infty} \leq B_{1} B_{2} \sum_{k=0}^{M} \sum_{l=1}^{N(m, k)}\left|a_{k l}(n)-1\right|, \quad n \geq M .
$$

If $\lim _{n \rightarrow \infty} a_{k l}(n)=1, k \in \mathbb{N}, l=1,2 \ldots, N(m, k)$, then $\lim _{n \rightarrow \infty}\left\|T_{n}(q)-q\right\|_{\infty}=0$ and there is an $N(\epsilon) \in \mathbb{N}$ such that

$$
\left\|T_{n}(q)-q\right\|_{\infty}<\epsilon, \quad n \geq N(\epsilon)
$$

Therefore,

$$
\begin{aligned}
\left\|T_{n}(f)-f\right\|_{\infty} & \leq\left\|T_{n}(f)-T_{n}(q)\right\|_{\infty}+\left\|T_{n}(q)-q\right\|_{\infty}+\|q-f\|_{\infty} \\
& \leq\left\|T_{n}(f-q)\right\|_{\infty}+2 \epsilon \\
& \leq C(f-q)\|f-q\|_{\infty}+2 \epsilon \\
& \leq(C(f-q)+2) \epsilon, \quad n \geq N(\epsilon),
\end{aligned}
$$

completing the proof of the theorem.

TheOREM 3.3. Let $f \in L^{p}\left(S^{m}\right)$ be a function fulfilling the following condition: if $q \in P\left(S^{m}\right)$ then there exists a constant $C:=C(f-q) \geq 0$ such that $\left\|T_{n}(f-q)\right\|_{p} \leq C(f-q) \| f-$ $q \|_{p}, n \in \mathbb{N}$. If $\lim _{n \rightarrow \infty} a_{k l}(n)=1, k \in \mathbb{N}, l=1,2, \ldots, N(m, k)$ then $\lim _{n \rightarrow \infty}\left\|T_{n}(f)-f\right\|_{p}=$ 0 . 
Proof. Let $\epsilon>0$. Since $C\left(S^{m}\right)$ is dense in $L^{p}\left(S^{m}\right)$, there exists a function $g \in C\left(S^{m}\right)$ such that $\|f-g\|_{p}<\epsilon / 2$. The Weierstrass approximation theorem provides a polynomial $q$ such that $\|q-g\|_{\infty}<\epsilon / 2$. Hence,

$$
\|f-q\|_{p} \leq\|f-g\|_{p}+\|g-q\|_{p} \leq \frac{\epsilon}{2}+\|g-q\|_{\infty}<\epsilon .
$$

As in the proof of the previous theorem, if $\lim _{n \rightarrow \infty} a_{k l}(n)=1, k \in \mathbb{N}, l=1,2, \ldots, N(m, k)$ then $\lim _{n \rightarrow \infty}\left\|T_{n}(q)-q\right\|_{\infty}=0$. Thus, the inequality $\left\|T_{n}(q)-q\right\|_{p} \leq\left\|T_{n}(q)-q\right\|_{\infty}$ implies that $\lim _{n \rightarrow \infty}\left\|T_{n}(q)-q\right\|_{p}=0$. Now, there exists $N(\epsilon) \in \mathbb{N}$ such that

$$
\left\|T_{n}(q)-q\right\|_{p}<\epsilon, \quad n \geq N(\epsilon) .
$$

Therefore,

$$
\begin{aligned}
\left\|T_{n}(f)-f\right\|_{p} & \leq\left\|T_{n}(f)-T_{n}(q)\right\|_{p}+\left\|T_{n}(q)-q\right\|_{p}+\|q-f\|_{p} \\
& \leq\left\|T_{n}(f-q)\right\|_{p}+2 \epsilon \\
& \leq C(f-q)\|f-q\|_{p}+2 \epsilon \\
& \leq(C(f-q)+2) \epsilon, \quad n \geq N(\epsilon) .
\end{aligned}
$$

This completes the proof.

Combining the previous three theorems we have the following.

Theorem 3.4. The sequence $\left\{T_{n}\right\}_{n \in \mathbb{N}}$ is an approximate identity in $X$ if and only if the following conditions hold:

(i) Given $f \in X$, there exists a constant $C_{f} \geq 0$ such that $\left\|T_{n}(f)\right\|_{X} \leq C_{f}\|f\|_{X}, n \in \mathbb{N}$;

(ii) $\lim _{n \rightarrow \infty} a_{k l}(n)=1, k \in \mathbb{N}, l=1,2, \ldots, N(m, k)$.

Next, we investigate the possibility of changing condition (i) by another involving the kernel $K_{n}$. This is suggested by Theorem 2.1.

Theorem 3.5. If $\lim _{n \rightarrow \infty} a_{k l}(n)=1, k \in \mathbb{N}, l=1,2, \ldots, N(m, k)$ and

$$
\sup _{n \in \mathbb{N}}\left(\sup \left\{\left\|K_{n}^{x}\right\|_{1}: x \in S^{m}\right\}\right)<\infty
$$

then $\left\{T_{n}\right\}_{n \in \mathbb{N}}$ is an approximate identity in $X$.

Proof. If $\sup \left\{\left\|K_{n}^{x}\right\|_{1}: x \in S^{m}\right\} \leq C, n \in \mathbb{N}$, for some positive constant $C$, not depending on $n$, then Theorem 2.4 implies that

$$
\left\|T_{n}(f)\right\|_{X} \leq C\|f\|_{X}, \quad n \in \mathbb{N}, f \in X .
$$

The previous theorem closes the proof.

The converse of this result holds in at least two cases.

Theorem 3.6. Let either $X=C\left(S^{m}\right)$ or $X=L^{1}\left(S^{m}\right)$. Then $\left\{T_{n}\right\}_{n \in \mathbb{N}}$ is an approximate identity in $X$ if and only if the following properties hold:

(i) $\sup _{n \in \mathbb{N}}\left(\sup \left\{\left\|K_{n}^{x}\right\|_{1}: x \in S^{m}\right\}\right)<\infty$;

(ii) $\lim _{n \rightarrow \infty} a_{k l}(n)=1, k \in \mathbb{N}, l=1, \ldots, N(m, k)$. 
332 Approximation on the sphere by weighted Fourier expansions

Proof. One implication is consequence of the previous theorem. As for the other, assume $\left\{T_{n}\right\}_{n \in \mathbb{N}}$ is an approximate identity in $X$. Theorem 3.1 yields the existence of a positive constant $C$ such that

$$
\left\|T_{n}(f)\right\|_{X} \leq C\|f\|_{X}, \quad f \in X, n \in \mathbb{N} .
$$

Hence,

$$
\left\|T_{n}\right\|_{X}=\sup _{f \in X \backslash\{0\}} \frac{\left\|T_{n}(f)\right\|_{X}}{\|f\|_{X}} \leq C, \quad n \in \mathbb{N} .
$$

Under the conditions in the statement of the theorem, Theorem 2.5 is applicable. Hence,

$$
\sup \left\{\left\|K_{n}^{x}\right\|_{1}: x \in S^{m}\right\}=\left\|T_{n}\right\|_{X} \leq C, \quad n \in \mathbb{N},
$$

and, therefore,

$$
\sup _{n \in \mathbb{N}}\left(\sup \left\{\left\|K_{n}^{x}\right\|_{1}: x \in S^{m}\right\}\right) \leq C .
$$

This takes care of condition (i). Condition (ii) follows directly from Theorem 3.1.

If $K_{n}$ is positive, that is, $K_{n}(x, y) \geq 0, x, y \in S^{m}$, then we can sharpen Theorem 3.6 as follows.

Theorem 3.7. Let either $X=C\left(S^{m}\right)$ or $X=L^{1}\left(S^{m}\right)$. Assume that every $K_{n}$ is positive. Then $\left\{T_{n}\right\}_{n \in \mathbb{N}}$ is an approximate identity in $X$ if and only if $\lim _{n \rightarrow \infty} a_{k l}(n)=1, k \in \mathbb{N}$, $l=1,2, \ldots, N(m, k)$.

Proof. It is sufficient to prove that (ii) implies (i) in Theorem 3.6. The positivity of $K_{n}$ and the orthonormality of the spherical harmonics yield

$$
\begin{aligned}
\left\|K_{n}^{x}\right\|_{1} & =\frac{1}{\sigma_{m}} \int_{S^{m}} K_{n}(x, y) d \sigma_{m}(y) \\
& =\frac{1}{\sigma_{m}} \int_{S^{m}} \sum_{k=0}^{n} \sum_{l=1}^{N(m, k)} a_{k l}(n) Y_{k l}(x) \overline{Y_{k l}(y)} d \sigma_{m}(y) \\
& =\sum_{k=0}^{n} \sum_{l=1}^{N(m, k)} a_{k l}(n) Y_{k l}(x) \frac{1}{\sigma_{m} Y_{01}(x)} \int_{S^{m}} Y_{01}(y) \overline{Y_{k l}(y)} d \sigma_{m}(y) \\
& =a_{01}(n), \quad x \in S^{m}, n \in \mathbb{N} .
\end{aligned}
$$

Hence,

$$
\sup _{x \in S^{m}}\left\|K_{n}^{x}\right\|_{1}=a_{01}(n), \quad n \in \mathbb{N} .
$$

Since $\lim _{n \rightarrow \infty} a_{01}(n)=1$, it follows

$$
\lim _{n \rightarrow \infty} \sup _{x \in S^{m}}\left\|K_{n}^{x}\right\|_{1}=\lim _{n \rightarrow \infty} a_{01}(n)=1
$$


Therefore, there exists a positive constant $C$ such that

$$
\sup _{n \in \mathbb{N}}\left(\sup \left\{\left\|K_{n}^{x}\right\|_{1}: x \in S^{m}\right\}\right) \leq C .
$$

Thus, the result follows from the previous theorem.

These are elementary consequences of the above results.

Theorem 3.8. Let either $X=C\left(S^{m}\right)$ or $X=L^{1}\left(S^{m}\right)$. If $\lim _{n \rightarrow \infty} a_{k l}(n)=1, k \in \mathbb{N}, l=1$, $\ldots, N(m, k)$, then the following assertions are equivalent:

(i) $\lim _{n \rightarrow \infty}\left\|T_{n}(f)-f\right\|_{X}=0, f \in X$;

(ii) there exists a $C \geq 0$ such that $\left\|T_{n}(f)\right\|_{X} \leq C\|f\|_{X}, f \in X, n \in \mathbb{N}$;

(iii) $\sup _{n \in \mathbb{N}}\left(\sup \left\{\left\|K_{n}^{x}\right\|_{1}: x \in S^{m}\right\}\right)<\infty$.

Theorem 3.9. The sequence $\left\{T_{n}\right\}_{n \in \mathbb{N}}$ is an approximate identity in $C\left(S^{m}\right)$ if and only if it is an approximate identity in $L^{1}\left(S^{m}\right)$.

If $\left\{T_{n}\right\}_{n \in \mathbb{N}}$ is an approximate identity in $C\left(S^{m}\right)$ then it is an approximate identity in $L^{p}\left(S^{m}\right), 1 \leq p<\infty$ (Theorems 3.5 and 3.6). Thus, it is quite obvious that the case $X=$ $C\left(S^{m}\right)$ is the most important among all.

We close the section, giving a method to construct approximate identities. It is based on a corresponding property of spherical convolution.

Theorem 3.10. Let $\left\{S_{n}\right\}_{n \in \mathbb{N}}$ be another sequence having a representation as in (1.9). If both $\left\{T_{n}\right\}_{n \in \mathbb{N}}$ and $\left\{S_{n}\right\}_{n \in \mathbb{N}}$ are approximate identities in $X$ then $\left\{T_{n} \circ S_{n}\right\}_{n \in \mathbb{N}}$ is an approximate identity in $X$.

Proof. Let $\left\{T_{n}\right\}_{n \in \mathbb{N}}$ be as in (1.9) and represent $\left\{S_{n}\right\}_{n \in \mathbb{N}}$ in the form

$$
S_{n}(f)=\frac{1}{\sigma_{m}} \sum_{i=0}^{n} \sum_{j=1}^{N(m, i)} c_{i j}(n) \hat{f}(i, j) Y_{i j}, \quad f \in X,
$$

with $c_{i j}(n) \in \mathbb{R}, i=0,1, \ldots, j=1,2, \ldots, N(m, i)$. Using (2.5), (2.6) and arranging we have that

$$
\begin{aligned}
\left(T_{n}\left(S_{n} f\right)\right)(x) & =\frac{1}{\sigma_{m}} \int_{S^{m}} K_{n}(x, y)\left(S_{n} f\right)(y) d \sigma_{m}(y) \\
& =\frac{1}{\sigma_{m}^{2}} \int_{S^{m}} \sum_{k=0}^{n} \sum_{l=1}^{N(m, k)} a_{k l}(n) Y_{k l}(x) \overline{Y_{k l}(y)} \sum_{i=0}^{n} \sum_{j=1}^{N(m, i)} c_{i j}(n) \hat{f}(i, j) Y_{i j}(y) d \sigma_{m}(y),
\end{aligned}
$$

whence

$$
\begin{aligned}
\left(T_{n}\left(S_{n} f\right)\right)(x) & =\frac{1}{\sigma_{m}} \sum_{k=0}^{n} \sum_{l=1}^{N(m, k)} \sum_{i=0}^{n} \sum_{j=1}^{N(m, i)} a_{k l}(n) c_{i j}(n) \hat{f}(i, j) Y_{k l}(x) \frac{1}{\sigma_{m}} \int_{S^{m}} Y_{i j}(y) \overline{Y_{k l}(y)} d \sigma_{m}(y) \\
& =\frac{1}{\sigma_{m}} \sum_{k=0}^{n} \sum_{l=1}^{N(m, k)} a_{k l}(n) c_{k l}(n) \hat{f}(k, l) Y_{k l}(x), \quad x \in S^{m}, f \in X .
\end{aligned}
$$


334 Approximation on the sphere by weighted Fourier expansions

If $\left\{T_{n}\right\}_{n \in \mathbb{N}}$ and $\left\{S_{n}\right\}_{n \in \mathbb{N}}$ are approximate identities in $X$, Theorem 3.1 implies that

$$
\lim _{n \rightarrow \infty} a_{k l}(n)=\lim _{n \rightarrow \infty} c_{k l}(n)=1, \quad k \in \mathbb{N}, l=1,2, \ldots, N(m, k)
$$

Hence,

$$
\lim _{n \rightarrow \infty} a_{k l}(n) c_{k l}(n)=1, \quad k \in \mathbb{N}, l=1,2, \ldots, N(m, k)
$$

That same theorem produces constants $C_{1}, C_{2}>0$ such that

$$
\left\|T_{n}(f)\right\|_{X} \leq C_{1}\|f\|_{X}, \quad\left\|S_{n}(f)\right\|_{X} \leq C_{2}\|f\|_{X}, \quad n \in \mathbb{N}, f \in X .
$$

Thus,

$$
\left\|T_{n}\left(S_{n} f\right)\right\|_{X} \leq C_{1}\left\|S_{n}(f)\right\|_{X} \leq C_{1} C_{2}\|f\|_{X}, \quad n \in \mathbb{N}, f \in X
$$

Now, Theorem 3.4 guarantees that $\left\{T_{n} \circ S_{n}\right\}_{n \in \mathbb{N}}$ is an approximate identity in $X$.

An important consequence of Theorem 3.10 is this: if $\left\{T_{n}\right\}_{n \in \mathbb{N}}$ is an approximate identity in $X$ and $k \in \mathbb{N}$ then $\left\{T_{n}^{k}\right\}_{n \in \mathbb{N}}$ is an approximate identity in $X$.

\section{Localized approximate identities}

In this section, we deal with the space $C\left(S^{m}\right)$ only and investigate approximation at one fixed point. The term localized refers to this and nothing else. To motivate the main definition in this section, let $\left\{T_{n}\right\}$ be as in (1.9) and assume that $\left\{T_{n}\right\}_{n \in \mathbb{N}}$ is an approximate identity in $C\left(S^{m}\right)$. Since

$$
\lim _{n \rightarrow \infty} T_{n} f(y)=f(y), \quad y \in S^{m}, f \in C\left(S^{m}\right)
$$

we have that

$$
\begin{aligned}
f(y) & =\lim _{n \rightarrow \infty} \frac{1}{\sigma_{m}} \sum_{k=0}^{n} \sum_{l=1}^{N(m, k)} a_{k l}(n) \hat{f}(k, l) Y_{k l}(y) \\
& =\lim _{n \rightarrow \infty} \frac{1}{\sigma_{m}} \int_{S^{m}} \sum_{k=0}^{n} \sum_{l=1}^{N(m, k)} a_{k l}(n) Y_{k l}(y) \overline{Y_{k l}(x)} f(x) d \sigma_{m}(x) \\
& =\lim _{n \rightarrow \infty} \frac{1}{\sigma_{m}} \int_{S^{m}}\left(\sum_{k=0}^{n} \sum_{l=1}^{N(m, k)} c_{k l}^{y}(n) \overline{Y_{k l}(x)}\right) f(x) d \sigma_{m}(x), \quad y \in S^{m}, f \in C\left(S^{m}\right),
\end{aligned}
$$

in which $c_{k l}^{y}(n):=a_{k l}(n) Y_{k l}(y)$. 
The definition can now be introduced. Let $y \in S^{m}$ and let $\left\{S_{n}^{y}\right\}_{n \in \mathbb{N}}$ be a sequence of complex functions defined on $S^{m}$ that depends on $y$. The sequence is called an approximate identity in $y$ if

$$
\lim _{n \rightarrow \infty} \frac{1}{\sigma_{m}} \int_{S^{m}} S_{n}^{y}(x) f(x) d \sigma_{m}(x)=f(y), \quad f \in C\left(S^{m}\right) .
$$

THEOREM 4.1. If $\left\{T_{n}\right\}_{n \in \mathbb{N}}$ is an approximate identity in $C\left(S^{m}\right)$ then the sequence $\left\{T_{n}^{y}\right\}_{n \in \mathbb{N}}$ given by

$$
T_{n}^{y}(x):=\sum_{k=0}^{n} \sum_{l=1}^{N(m, k)} c_{k l}^{y}(n) \overline{Y_{k l}(x)}, \quad x \in S^{m},
$$

in which $c_{k l}^{y}(n)=a_{k l}(n) Y_{k l}(y), k=0,1, \ldots, l=1,2, \ldots, N(m, k)$, is an approximate identity in $y$.

In what follows we will seek for conditions in order that a general sequence $\left\{T_{n}^{y}\right\}_{n \in \mathbb{N}}$ as in (4.4), but with arbitrary coefficients $c_{k l}^{y}(n)$, be an approximate identity in $y$. We begin computing the norm of the operator generated by (4.4).

Theorem 4.2. Let $L_{n}^{y}$ denote the linear functional on $C\left(S^{m}\right)$ given by

$$
L_{n}^{y}(f)=\frac{1}{\sigma_{m}} \int_{S^{m}} T_{n}^{y}(x) f(x) d \sigma_{m}(x), \quad f \in C\left(S^{m}\right) .
$$

Then $L_{n}^{y}$ is continuous and $\left\|L_{n}^{y}\right\|_{C\left(S^{m}\right)}=\left\|T_{n}^{y}\right\|_{1}$.

Proof. This is standard but we include a sketch of the proof for the sake of completeness and because the same arguments are present in the proof of the next result. If $f \in C\left(S^{m}\right)$, then

$$
\begin{aligned}
\left|L_{n}^{y}(f)\right| & =\left|\frac{1}{\sigma_{m}} \int_{S^{m}} T_{n}^{y}(x) f(x) d \sigma_{m}(x)\right| \\
& \leq \frac{1}{\sigma_{m}} \int_{S^{m}}\left|T_{n}^{y}(x)\right||f(x)| d \sigma_{m}(x) \\
& \leq\|f\|_{\infty}\left\|T_{n}^{y}\right\|_{1}, \quad f \in C\left(S^{m}\right),
\end{aligned}
$$

and, consequently,

$$
\left\|L_{n}^{y}\right\|_{C\left(S^{m}\right)}=\sup \left\{\left|L_{n}^{y} f\right|:\|f\|_{\infty} \leq 1\right\} \leq\left\|T_{n}^{y}\right\|_{1} .
$$

On the other hand, since $F:=\left\{x \in S^{m}: T_{n}^{y}(x)=0\right\}$ is closed, given an $\epsilon>0$, there exists an open set $O_{\epsilon} \subset S^{m}$ such that $F \subset O_{\epsilon}$ and

$$
\frac{1}{\sigma_{m}} \int_{O_{\epsilon}}\left|T_{n}^{y}(x)\right| d \sigma_{m}(x)<\epsilon
$$

Since $S^{m}$ is a normal space, we can find a continuous function $g_{\epsilon}: S^{m} \rightarrow[0,1]$ such that $g_{\epsilon}(x)=0, x \in F$ and $g_{\epsilon}(x)=1, x \in S^{m} \backslash O_{\epsilon}$. Now, it is promptly seen that the function $h_{\epsilon}$ defined by $h_{\epsilon}(x)=g_{\epsilon} \operatorname{sign} \overline{T_{n}^{y}(x)}, x \in S^{m}$ is continuous and $\left\|h_{\epsilon}\right\|_{\infty} \leq 1$. Here, 
336 Approximation on the sphere by weighted Fourier expansions

$\operatorname{sign} z=z /|z|, z \neq 0$ and $\operatorname{sign} 0=0$. In addition,

$$
\begin{aligned}
L_{n}^{y}\left(h_{\epsilon}\right) & =\frac{1}{\sigma_{m}} \int_{S^{m}} T_{n}^{y}(x) g_{\epsilon}(x) \operatorname{sign} \overline{T_{n}^{y}(x)} d \sigma_{m}(x) \\
& \geq \frac{1}{\sigma_{m}} \int_{S^{m} \backslash O_{\epsilon}}\left|T_{n}^{y}(x)\right| d \sigma_{m}(x) \geq\left\|T_{n}^{y}\right\|_{1}-\epsilon .
\end{aligned}
$$

Thus, $\left\|L_{n}^{y}\right\|_{C\left(S^{m}\right)} \geq\left\|T_{n}^{y}\right\|_{1}-\epsilon$. This completes the proof.

The existence of localized approximate identities follows from the results in the previous sections along with the comments we have made at the beginning of this section. Theorem 4.3 provides an independent proof of this same result.

Theorem 4.3. Given $y \in S^{m}$, there always exists an approximate identity in $y$.

Proof. Let $y \in S^{m}$ and let $O_{n}^{y}$ be the set formed by the elements of $S^{m}$ which are within $1 / n$ of $y$. Since $O_{n}^{y}$ is open in $\mathbb{R}^{m+1}$, Urysohn's lemma implies that we can find a sequence $\left\{g_{n}^{y}\right\}_{n \in \mathbb{N}} \subset C\left(S^{m}\right)$ such that $g_{n}^{y}(x) \geq 0, x \in S^{m}, g_{n}^{y}(x)=0$ for $x \in S^{m} \backslash O_{n}^{y}$ and

$$
\frac{1}{\sigma_{m}} \int_{S^{m}} g_{n}^{y}(x) d \sigma_{m}(x)=1 .
$$

On the other hand, the Weierstrass approximation theorem allows us to find a family $\left\{q_{n}^{y}\right\}_{n \in \mathbb{N}}$ of polynomials in $m+1$ variables such that

$$
\left\|g_{n}^{y}-q_{n}^{y}\right\|_{\infty}<\frac{1}{n}, \quad n \in \mathbb{N} .
$$

Let $f \in C\left(S^{m}\right)$ and fix $\epsilon>0$. From the continuity of $f$, we can find an $n_{0}=n_{0}(\epsilon) \in \mathbb{N}$ such that

$$
|f(y)-f(x)|<\epsilon, \quad x \in O_{n_{0}}^{y}
$$

In addition, we can choose $n_{1} \geq n_{0}$ such that

$$
|f(y)-f(x)|<\epsilon, \quad x \in O_{n_{1}}^{y}
$$

and $\|f\|_{\infty}<\epsilon n_{1}$. Since

$$
\begin{aligned}
\left|f(y)-\frac{1}{\sigma_{m}} \int_{S^{m}} q_{n}^{y}(x) f(x) d \sigma_{m}(x)\right| \leq & \left|\frac{1}{\sigma_{m}} \int_{S^{m}}[f(y)-f(x)] g_{n}^{y}(x) d \sigma_{m}(x)\right| \\
& +\left|\frac{1}{\sigma_{m}} \int_{S^{m}}\left[g_{n}^{y}(x)-q_{n}^{y}(x)\right] f(x) d \sigma_{m}(x)\right| \\
\leq & \frac{1}{\sigma_{m}} \int_{S^{m}}|f(y)-f(x)| g_{n}^{y}(x) d \sigma_{m}(x) \\
& +\frac{1}{\sigma_{m}} \int_{S^{m}}\left|g_{n}^{y}(x)-q_{n}^{y}(x)\right||f(x)| d \sigma_{m}(x),
\end{aligned}
$$


we deduce that

$$
\begin{aligned}
& \left|f(y)-\frac{1}{\sigma_{m}} \int_{S^{m}} q_{n}^{y}(x) f(x) d \sigma_{m}(x)\right| \\
& \quad \leq \frac{1}{\sigma_{m}} \int_{O_{n}^{y}}|f(y)-f(x)| g_{n}^{y}(x) d \sigma_{m}(x)+\left\|g_{n}^{y}-q_{n}^{y}\right\|_{\infty}\|f\|_{\infty} \\
& \leq \epsilon \frac{1}{\sigma_{m}} \int_{O_{n}^{y}} g_{n}^{y}(x) d \sigma_{m}(x)+\frac{\|f\|_{\infty}}{n} \\
& \leq 2 \epsilon, \quad n \geq n_{1} .
\end{aligned}
$$

Since $q_{n}^{y}$ is representable in the form

$$
q_{n}^{y}(x)=\sum_{k=0}^{M_{n}} \sum_{l=1}^{N(m, k)} c_{k l}^{y}(n) \overline{Y_{k l}(x)}, \quad x \in S^{m},
$$

for some $M_{n}$, the result follows.

The main result of this section is as follows.

Theorem 4.4. Let $y \in S^{m}$. The sequence $\left\{T_{n}^{y}\right\}_{n \in \mathbb{N}}$ is an approximate identity in $y$ if and only if the following conditions hold:

(i) There exists a positive constant $C$ such that $\left\|T_{n}^{y}\right\|_{1} \leq C, n \in \mathbb{N}$;

(ii) $\lim _{n \rightarrow \infty} c_{k l}^{y}(n)=Y_{k l}(y), k \in \mathbb{N}, l=1,2, \ldots, N(m, k)$.

Proof. Assume $\left\{T_{n}^{y}\right\}_{n \in \mathbb{N}}$ is an approximate identity in $y$. Since $\lim _{n \rightarrow \infty} L_{n}^{y}(f)=f(y), f \in$ $C\left(S^{m}\right)$, it follows that each sequence $\left\{L_{n}^{y}(f)\right\}_{n \in \mathbb{N}}$ is bounded. Hence, for every $f \in C\left(S^{m}\right)$, we can find a constant $C_{f} \geq 0$ such that

$$
\sup \left\{\left|L_{n}^{y}(f)\right|: n \in \mathbb{N}\right\} \leq C_{f} .
$$

The Uniform Boundedness Principle implies that

$$
\left\|T_{n}^{y}\right\|_{1}=\left\|L_{n}^{y}\right\|_{C\left(S^{m}\right)} \leq C, \quad n \in \mathbb{N}
$$

for some $C \geq 0$. It is an easy matter to verify that $C$ is in fact positive. Since

$$
\begin{aligned}
L_{n}^{y}\left(Y_{\mu \nu}\right) & =\frac{1}{\sigma_{m}} \int_{S^{m}} T_{n}^{y}(x) Y_{\mu \nu}(x) d \sigma_{m}(x) \\
& =\sum_{k=0}^{\mu} \sum_{l=1}^{N(m, k)} c_{k l}^{y}(n)\left(\frac{1}{\sigma_{m}} \int_{S^{m}} Y_{\mu \nu}(x) \overline{Y_{k l}(x)} d \sigma_{m}(x)\right) \\
& =c_{\mu \nu}^{y}(n), \quad n \geq \mu .
\end{aligned}
$$

then

$$
\lim _{n \rightarrow \infty} c_{\mu \nu}^{y}(n)=\lim _{n \rightarrow \infty} L_{n}^{y}\left(Y_{\mu \nu}\right)=Y_{\mu \nu}(y), \quad \mu \in \mathbb{N}, \nu=1,2, \ldots, N(m, \mu)
$$


338 Approximation on the sphere by weighted Fourier expansions

Conversely, assume that (i) and (ii) hold. Let $f \in C\left(S^{m}\right)$ and fix $\epsilon>0$. By the Weierstrass approximation theorem we can find a polynomial $q$ in $m+1$ variables so that $\|q-f\|_{\infty}<\epsilon$. Writing $q$ in the form

$$
q=\sum_{\mu=0}^{M} \sum_{\nu=1}^{N(m, \mu)} r_{\mu \nu} Y_{\mu \nu}, \quad r_{\mu \nu} \in \mathbb{C}
$$

and observing that $L_{n}^{y}(q)=\sum_{k=0}^{M} \sum_{l=1}^{N(m, k)} c_{k l}^{y}(n) r_{k l}, n \geq M$, condition (ii) implies that

$$
\lim _{n \rightarrow \infty} L_{n}^{y}(q)=\lim _{n \rightarrow \infty} \sum_{k=0}^{M} \sum_{l=1}^{N(m, k)} c_{k l}^{y}(n) r_{k l}=\sum_{k=0}^{M} \sum_{l=1}^{N(m, k)} r_{k l} Y_{k l}(y)=q(y) .
$$

Choosing $N(\epsilon)$ so that

$$
\left|L_{n}^{y}(q)-q(y)\right|<\epsilon, \quad n \geq N(\epsilon)
$$

we can use condition (i) to reach

$$
\begin{aligned}
\left|L_{n}^{y}(f)-f(y)\right| & \leq\left|L_{n}^{y}(f)-L_{n}^{y}(q)\right|+\left|L_{n}^{y}(q)-q(y)\right|+|q(y)-f(y)| \\
& \leq\left|L_{n}^{y}(f-q)\right|+\epsilon+\|q-f\|_{\infty} \\
& <\left\|L_{n}^{y}\right\|_{C\left(S^{m}\right)}\|(f-q)\|_{\infty}+2 \epsilon \\
& =\left\|T_{n}^{y}\right\|_{1}\|(f-q)\|_{\infty}+2 \epsilon \\
& <(C+2) \epsilon, \quad n \geq N(\epsilon) .
\end{aligned}
$$

This completes the proof.

We close the paper stating a theorem that includes a positiveness hypothesis on $T_{n}^{y}$.

Theorem 4.5. Let $y \in S^{m}$ and assume that every $T_{n}^{y}$ is positive. Then $\left\{T_{n}^{y}\right\}_{n \in \mathbb{N}}$ is an approximate identity in $y$ if and only if $\lim _{n \rightarrow \infty} c_{k l}^{y}(n)=Y_{k l}(y), k \in \mathbb{N}, l=1,2, \ldots, N(m, k)$.

Proof. The positivity of $T_{n}^{y}$ and the orthonormality of the spherical harmonics yield

$$
\begin{aligned}
\left\|T_{n}^{y}\right\|_{1} & =\frac{1}{\sigma_{m}} \int_{S^{m}} T_{n}^{y}(x) d \sigma_{m}(x) \\
& =\frac{1}{\sigma_{m}} \int_{S^{m}} \sum_{k=0}^{n} \sum_{l=1}^{N(m, k)} c_{k l}^{y}(n) \overline{Y_{k l}(x)} d \sigma_{m}(x) \\
& =\sum_{k=0}^{n} \sum_{l=1}^{N(m, k)} c_{k l}^{y}(n) \frac{1}{\sigma_{m} Y_{01}(y)} \int_{S^{m}} Y_{01}(x) \overline{Y_{k l}(x)} d \sigma_{m}(x) \\
& =\frac{c_{01}^{y}(n)}{Y_{01}(y)} .
\end{aligned}
$$

Hence, condition (ii) in Theorem 4.4 implies condition (i) there. The rest follows. 


\section{References}

[1] S. Axler, P. Bourdon, and W. Ramey, Harmonic Function Theory, Graduate Texts in Mathematics, vol. 137, Springer, New York, 1992.

[2] P. J. Davis, Interpolation and Approximation, Dover, New York, 1975, Republication, with minor corrections, of the 1963 original, with a new preface and bibliography.

[3] G. B. Folland, Real Analysis. Modern Techniques and Their Applications, 2nd ed., Pure and Applied Mathematics (New York), A Wiley-Interscience Publication. John Wiley \& Sons, New York, 1999.

[4] W. Freeden, T. Gervens, and M. Schreiner, Constructive Approximation on the Sphere. With Applications to Geomathematics, Numerical Mathematics and Scientific Computation, The Clarendon Press, Oxford University Press, New York, 1998.

[5] W. Freeden, O. Glockner, and R. Litzenberger, A general Hilbert space approach to wavelets and its application in geopotential determination, Numer. Funct. Anal. Optim. 20 (1999), no. 9$10,853-879$.

[6] W. Freeden and K. Hesse, On the multiscale solution of satellite problems by use of locally supported kernel functions corresponding to equidistributed data on spherical orbits, Studia Sci. Math. Hungar. 39 (2002), no. 1-2, 37-74.

[7] H. Groemer, Geometric Applications of Fourier Series and Spherical Harmonics, Encyclopedia of Mathematics and Its Applications, vol. 61, Cambridge University Press, Cambridge, 1996.

[8] V. A. Menegatto, Approximation by spherical convolution, Numer. Funct. Anal. Optim. 18 (1997), no. 9-10, 995-1012.

[9] V. Michel, A multiscale approximation for operator equations in separable Hilbert spaces—case study: Reconstruction and description of the Earth's interior, Habilitation thesis, Shaker, Aachen, 2002.

[10] - Regularized wavelet-based multiresolution recovery of the harmonic mass density distribution from data of the Earth's gravitational field at satellite height, Inverse Problems 21 (2005), no. 3, 997-1025.

[11] M. Morimoto, Analytic Functionals on the Sphere, Translations of Mathematical Monographs, vol. 178, American Mathematical Society, Rhode Island, 1998.

[12] C. Müller, Spherical Harmonics, Lecture Notes in Mathematics, vol. 17, Springer, Berlin, 1966.

[13] Analysis of Spherical Symmetries in Euclidean Spaces, Applied Mathematical Sciences, vol. 129, Springer, New York, 1998.

[14] M. Reimer, Constructive Theory of Multivariate Functions. With an Application to Tomography, Bibliographisches Institut, Mannheim, 1990.

V. A. Menegatto: Departamento de Matemática, Instituto de Ciências Matemáticas de Computação, Universidade de São Paulo - São Carlos, Caixa Postal 668, 13560-970 São Carlos SP, Brazil

E-mail address: menegatt@icmc.usp.br

A. C. Piantella: Departamento de Matemática, Instituto de Ciências Matemáticas de Computação, Universidade de São Paulo - São Carlos, Caixa Postal 668, 13560-970 São Carlos SP, Brazil

E-mail address: anacarla@icmc.usp.br 


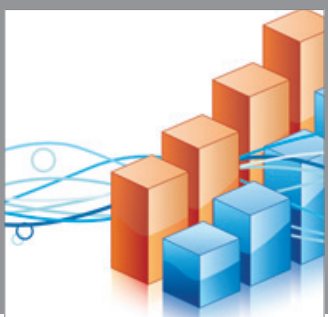

Advances in

Operations Research

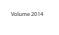

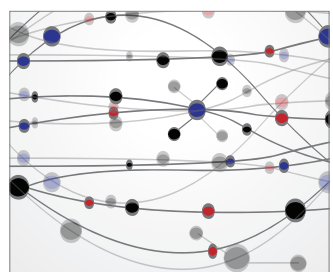

\section{The Scientific} World Journal
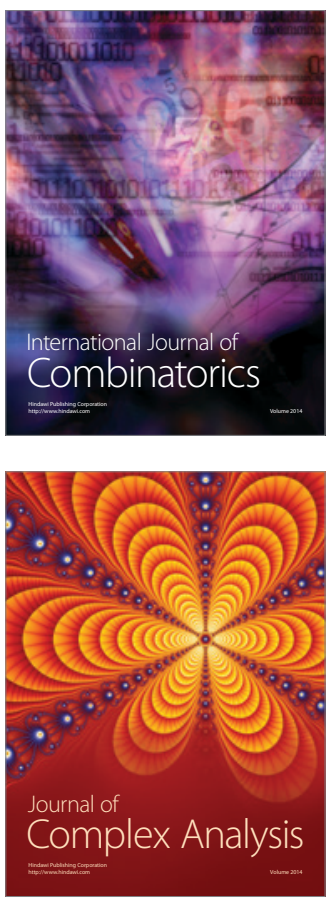

International Journal of

Mathematics and

Mathematical

Sciences
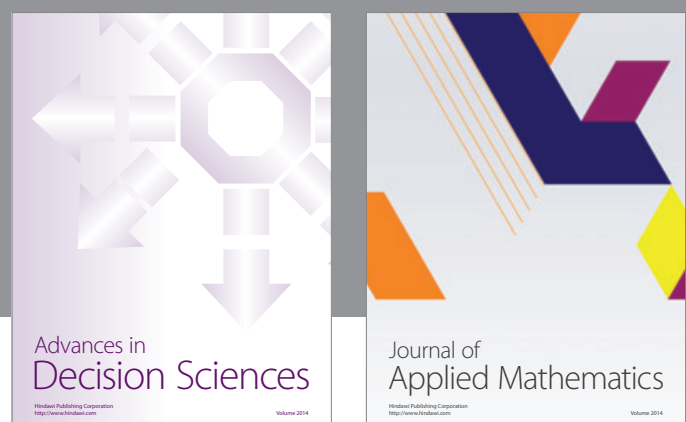

Journal of

Applied Mathematics
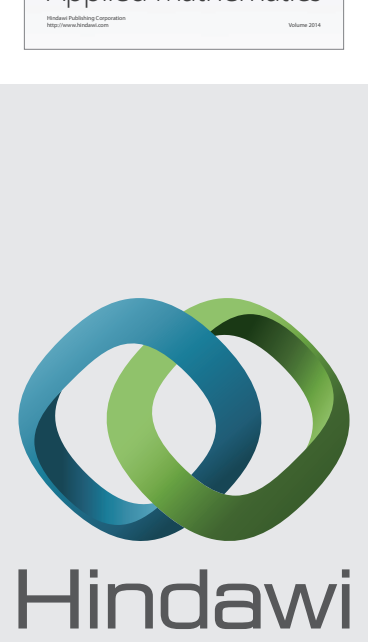

Submit your manuscripts at http://www.hindawi.com
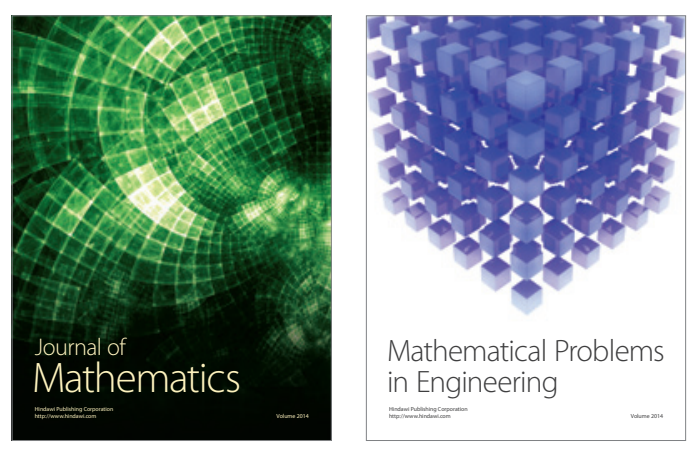

Mathematical Problems in Engineering
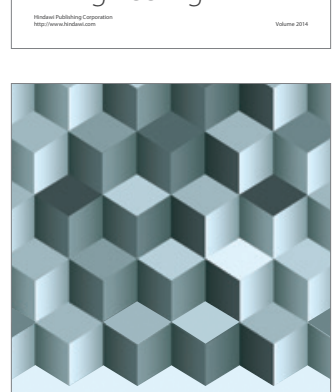

Journal of

Function Spaces
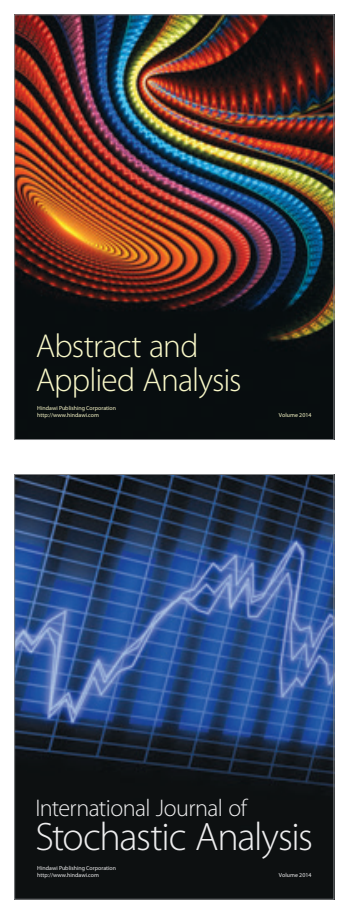

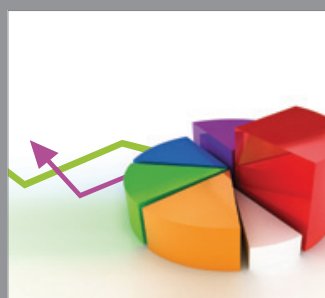

ournal of

Probability and Statistics

Promensencen
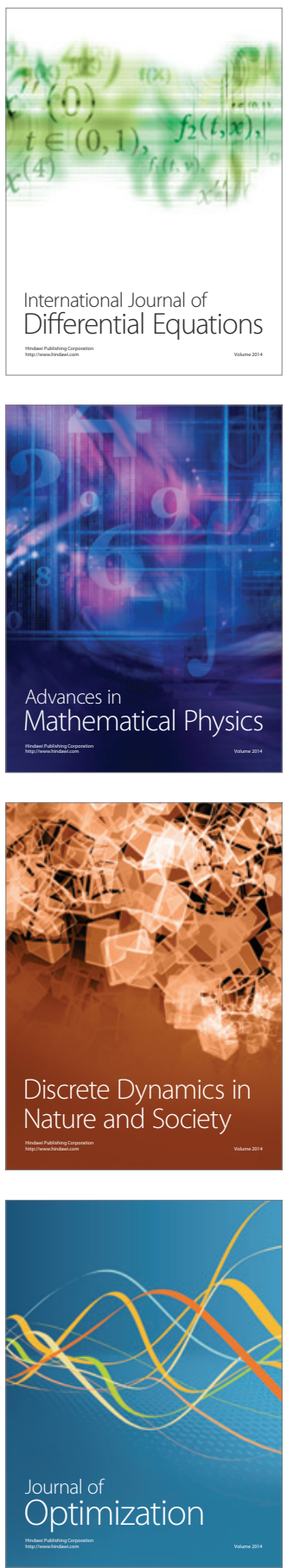